\title{
Hyperbolic metamaterials for terahertz applications
}

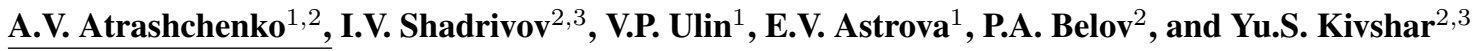 \\ ${ }^{1}$ Ioffe Physical-Technical Institute, Russian Academy of Sciences, St. Petersburg 194021, Russia \\ ${ }^{2}$ National Research University of Information Technologies, Mechanics and Optics, St. Petersburg 197101, \\ Russia \\ ${ }^{3}$ Nonlinear Physics Center, Research School of Physics and Engineering, Australian National University, \\ Canberra ACT 0200, Australia \\ astron86@mail.ru
}

\begin{abstract}
We develop a method for fabricating hyperbolic metamaterials for terahertz (THz) applications. We prepare a porous silicon matrix with a triangular lattice of holes, which we fill with copper by means of electrochemical deposition. We study transmission properties of this wire medium using THz time-domain spectroscopy, and demonstrate hyperbolic media properties.
\end{abstract}

\section{INTRODUCTION}

Numerous theoretical and numerical studies show exotic properties of hyperbolic metamaterials based on a wire medium [1]. Experimentally, wire media have been realised for microwave and optical frequencies, however at $\mathrm{THz}$ frequency it is still a challange to make such structues. For fabrication of wire metamaterials operating at $\mathrm{THz}$, infrared and visible frequencies, the most promising techniques are based on self-organization processes, which determine the arrangement and structure of metal wires. An example of such technology is the method of electrochemical etching, which allows fabrication of porous matrix of semiconductors $[2,3]$ and dielectrics [4]. The pores of matrices can be filled with different materials, including metals. This makes such matrices a basis for many composite dielectric-dielectric, metal-dielectric, metal-semiconductor and semiconductor-dielectric structures. The matrices can be fabricated with different hole sizes from several nanometres to several microns with a lattice constant ranging from tens of nanometres to several microns.

In this work, we fabricate large area macroporous matrix with a triangular lattice in lightly doped silicon and filled pores with copper using electrochemical deposition. As a result, the this medium exhibits hyperbolic media properties in the terahertz frequency range.

\section{FABRICATION OF THE WIRE MEDIUM BASED ON MACROPOROUS SI}

To fabricate a triangular lattice of macropores in n-type $\mathrm{Si}(100)$ wafer of the thikness of $250 \mu \mathrm{m}$, and resistivity of $\rho=5 \Omega \cdot \mathrm{cm}$, we use a photoelectrochemical etching technique [5,6]. Employing this method, we fabricate a macroporous matrix with through pores (of the same lenght as the substrate thickness, $250 \mu \mathrm{m}$ ) with the pore diameter of $2 \mu \mathrm{m}$ and lattice constant of $4 \mu \mathrm{m}$ (see Fig. 1a). To create a wire medium, we use the method of electrochemical deposition of metals [7]. First, one side of the macroporous matrix is covered by a gold layer using vacuum deposition, so that the whole surface including pores is covered by gold. Then, we performe the metal deposition of copper for 3000 minutes of an alcoholic solution of $\mathrm{CuCl}_{2}$ at the potential of $-1 \mathrm{~V}$ in the potentiostatic regime. Next, gold is removed by nitro-hydrochloric acid. The filling fraction of the macroporous matrix is then measured by gravimetric method, and it iss found to be approximately $70 \%$. As a result, we create the wire medium (wire diameter of $2 \mu \mathrm{m}$, length of $250 \mu \mathrm{m}$, lattice constant of $4 \mu \mathrm{m}$ ) made of copper enclosed in the matrix of lightly doped Si (see schematics in Fig. 1b). Surface of the wire medium is shown in Figure 1c. The manufactured sample area is $2.5 \mathrm{~cm}$ by $2.5 \mathrm{~cm}$. 

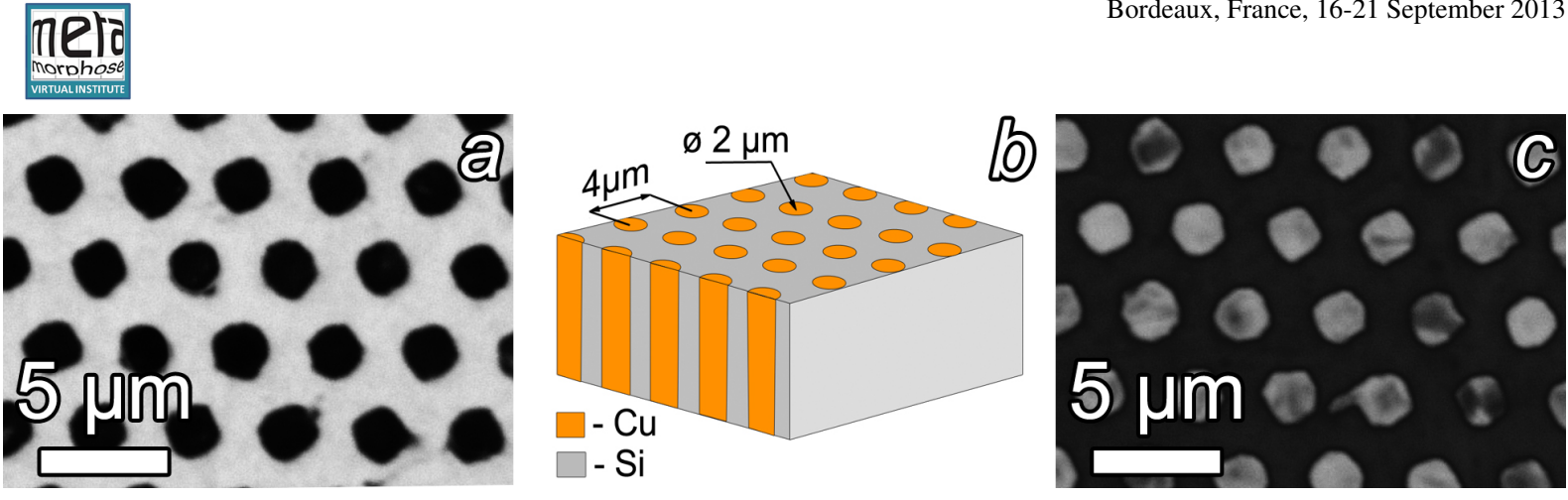

Fig. 1: Wire metamaterial based on a composite $\mathrm{Si}-\mathrm{Cu}$. a) Optical microscope image of the surface of the macroporous Si matrix. b) Schematic view of the wire metamaterial comprised of copper wires (diameter of $2 \mu \mathrm{m}$ ) forming the triangular lattice inside the Si matrix, with the lattice constant of $4 \mu \mathrm{m}$. c) Holes filled with copper are seen in the dark field image.

\section{EXPERIMENTAL CHARACTERIZATION}

Characterization of the THz response of the fabricated wire medium is performed using EKSPLA THz timedomain spectroscopy setup (THz-TDS). Transmission spectra of the wire medium are mesuared for different angles of incidence (deviation from the normal of 0, 5, 10 and 15 degrees). Measurement of the transmission spectra for larger angles is not possible in our setup due to a dramatic increase of absorbtion of $\mathrm{THz}$ waves in the wire medium, because of the increase of the electric field component directed along the wires. As a reference, we also mesuare the transmission spectra for the lightly doped $\mathrm{Si}$ substrate and for the macroporous matrix without copper infiltration. All measured spectra are normalised to the free-space transmission.

Assuming purely dielectric response of the metamaterial, we use measured complex transmission data for retreiving the index of refraction of the composite medium, by numerically inverting the Fresnel equations. The intensity $\mathbf{I}$ of the $\mathrm{THz}$ radiation transmitted through the wire medium can be found as

$$
I=I_{0}\left[\cosh ^{2} \chi d+\left(\frac{\chi^{2}-\alpha^{2}}{2 \chi \alpha}\right)^{2} \sinh ^{2} \chi d\right]^{-1}, \chi=\frac{\omega}{c} \sqrt{n_{1}^{2} \sin ^{2} \varphi-n_{2}^{2}}, \quad \alpha=\frac{\omega}{c} n_{1} \cos \varphi
$$

where $d$ is the thickness of the sample $(250 \mu \mathrm{m}), \varphi$ is the angle of incidence, $n_{1}$ and $n_{2}$ are the refractive indices of the wire medium and air, respectively, $\omega$ is angular frequency, and $c$ is the speed of light.

To test our extraction procedure, we measure the properties of the substrate and of the porous matrix. The refractive index of the lightly doped Si obtained from the analysis of the transmission spectra is found to be approximately 3.4, which is consistent with the values found in literature [8]. The refractive index of the macroporous matrix is around 2.67 which corresponds to the effective refractive index calculated from Maxwell-Garnett model [9].

The results of the retreived refractive index using Fresnel formula for the wire medium are shown in Fig. 2a. The dependence of the refractive index on the angle of incidence is also calculated using interference fringes at $1.4 \mathrm{THz}$, and it is shown in Figure 2b. Lower accuracy of determening of the refrective index is due to the difficulty of identifying the peaks of the interference, as compared to the calculations using Fresnel formula.

For the normal incidence of the $\mathrm{THz}$ waves, the refractive index is equal to the refractive index of the matrix medium ( $\mathrm{Si}$ ). This result is consistent with the theoretical analysis presented in Ref. [10]. We observe an increase of the refractive index with the increase of the angle of incidence. This is due to the fact that the wire medium is a uniaxial material, and with the propagation at an arbitrary angle the effective index becomes dependent on all components of the dielectric tensor. In this case, the change of the angle involves excitation of the currents in the wires by the component of the electric field directed along the wires. We show that this effect is a clear indication that our sample shows the properties of an anysotropic medium in the form of a hyperbolic material [1]. At the same time, with the increase of the refractive index with angle, both the reflection and absorbtion in the material grow, making it hard to characterise the sample. 


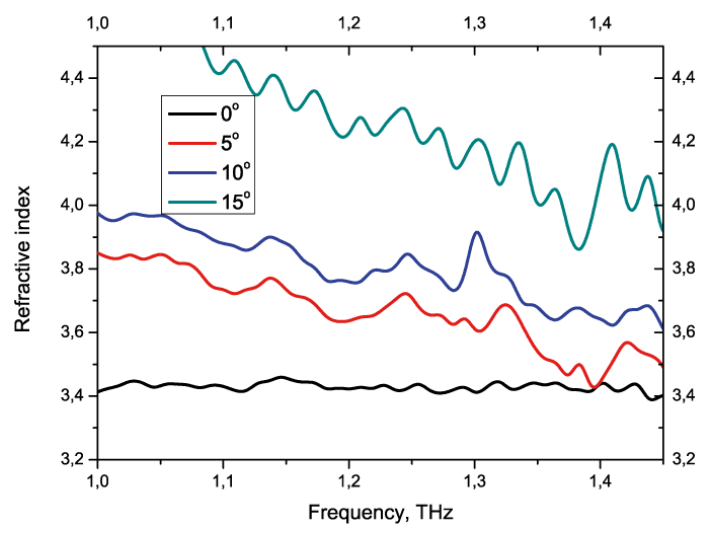

a)

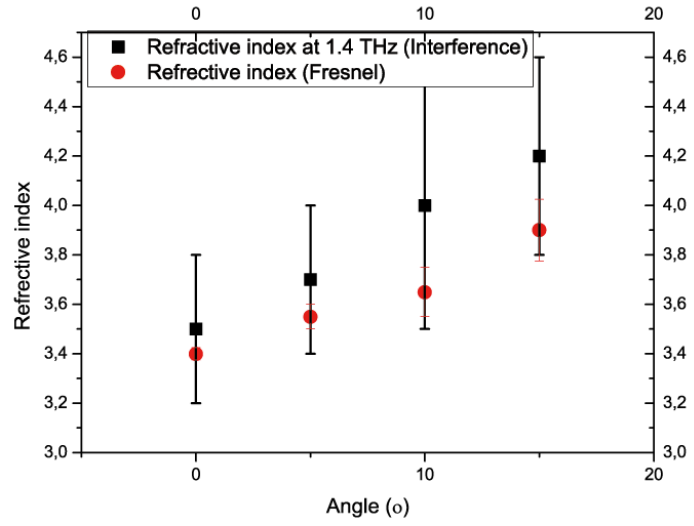

b)

Fig. 2: Effective refractive index of the wire metamaterial. (a) Frequency dependence of the refractive index calculated by inverting the Fresnel equations. (b) Angular dependence of the refractive index at $1.4 \mathrm{THz}$ calculated from the interference fringes and compared to that extracted from the Fresnel equations.

\section{CONCLUSIONS}

We have fabricated large scale terahertz wire metamaterial by employing the readily available electrochemical methods. The wire medium is based on the macroporous matrix of lightly doped Si whose pores are filled with copper. We have studied experimentally the properties of this medium such as the change of the refractive index as a function of angle. Such samples fabricated by the electrochemical methods will allow to access experimentally many nontrivial properties of the hyperbolic metamaterials predicted theoretically, including subwavelength imaging, magnification of subwavelength images, ultra-high Purcell factor, and others.

\section{ACKNOWLEDGEMENT}

This work has been supported by the RFBR (project 12-02-31439-mol-a) and by the Ministry of Education and Science of the Russian Federation (project 14.132.21.1403). The authors are indebted to Yu.M. Zadiranov for the gold deposition.

\section{REFERENCES}

[1] C.R. Simovski, P.A. Belov, A.V. Atrashchenko, Yu.S. Kivshar, "Wire Metamaterials: Physics and Applications," Advanced Materials, vol. 24, issue 31, pp. 42294248, 2012.

[2] V.P. Ulin and S.G. Konnikov, "Electrochemical pore formation mechanism in IIIV crystals (Part I)," Semiconductors, vol. 41, issue 7, pp. 832-844, 2007.

[3] H. Föll, M. Leisner, A. Cojocaru, and J. Carstensen, "Macroporous semiconductors," Materials, vol. 3, issue 5, pp. 3006$3076,2010$.

[4] A. Eftekhari, Nanostructured Materials in Electrochemistry. Chap. Highly Ordered Anodic Porous Alumina Formation by Self-Organized Anodizing, WILEY-VCH Verlag GmbH \& Co. KGaA, 2008.

[5] V. Lehmann and U. Gruning, "The limits of macropore array fabrication," Thin Solid Films, vol. 297, issue 12, pp. 13-17, 1997.

[6] E.V. Astrova, T.N. Borovinskaya, V.A. Tolmachev, and T.S. Perova, "Technique for patterning macroporous silicon and the fabrication of bars of 2D photonic crystals with vertical walls," Semiconductors, vol. 38, No. 9, pp. 1088-1091, 2004.

[7] H. Föll, M.-D. Gerngro $\beta$, A. Cojocaru, M. Leisner, J. Bahr, and J. Carstensen, "How to make single small holes with large aspect ratios," Phys. Status Solidi RRL, vol. 3, No. 2-3, pp. 55-57, 2009.

[8] J. Dai, J. Zhang, W. Zhang, and D. Grischkowsky, "Terahertz time-domain spectroscopy characterization of the farinfrared absorption and index of refraction of high-resistivity, float-zone silicon," J. Opt. Soc. Am. B, vol. 21, issue 7, pp. 1379-1386, 2004.

[9] J.C. Maxwell-Garnett, "Colours in metal glasses and in metallic films," Philos. Trans. R. Soc., vol. 203, pp. 385-420, 1904.

[10] S.J. Tyo, "A class of artificial materials isorefractive with free space," IEEE Transactions on Antennas and Propagation, vol. 51, No. 5, pp. 1093-1099, 2003. 